\title{
Impact of epidemiological characteristics of supratentorial gliomas in adults brought about by the 2016 world health organization classification of tumors of the central nervous system
}

\author{
Haihui Jiang ${ }^{1, *}$, Yong Cui 2,3,4,*, Junmei Wang ${ }^{5}$, Song Lin ${ }^{2,3,4}$ \\ ${ }^{1}$ Department of Neurosurgery, First Hospital of Tsinghua University, Beijing, China \\ ${ }^{2}$ Department of Neurosurgery, Beijing Tiantan Hospital, Capital Medical University, Beijing, China \\ ${ }^{3}$ China National Clinical Research Center for Neurological Diseases, Beijing, China \\ ${ }^{4}$ Beijing Institute for Brain Disorders and Beijing Key Laboratory of Brain Tumor, Beijing, China \\ ${ }^{5}$ Department of Pathology, Beijing Neurosurgical Institute, Beijing, China \\ *These authors have contributed equally to this work \\ Correspondence to: Song Lin, email: linsong2005@126.com
}

Keywords: gliomas, classification, epidemiology, impact

Received: July 27, $2016 \quad$ Accepted: November 07, $2016 \quad$ Published: November 24, 2016

\section{ABSTRACT}

The latest World Health Organization (WHO) classification of tumors of the central nervous system (CNS) integrates both histological and molecular features in the definition of diagnostic entities. This new approach enrolls novel entities of gliomas. In this study, we aimed to reveal the epidemiological characteristics, including age at diagnosis, gender ratio, tumor distribution and survival, of these new entities. We retrospectively reclassified 1210 glioma samples according to the 2016 CNS WHO diagnostic criteria. In our cohort, glioblastoma multiforme (GBM) with wildtype isocitrate dehydrogenase (IDH) was the most common malignant tumor in the brain. Almost all gliomas were more prevalent in males, especially in the cluster of WHO grade III gliomas and IDH-wildtype GBM. Age at diagnosis was directly proportional to tumor grade. With respect to the distribution by histology, we found that gliomas concurrent with IDH-mutant and 1p/19q-codeleted or with single IDHmutant were mainly distributed in frontal lobe, while those with IDH-wildtype were dominant in temporal lobe. Lesions located in insular lobe were more likely to be IDH-mutant astrocytoma. In summary, our results elucidated the epidemiological characteristics as well as the regional constituents of these new gliomas entities, which could bring insights into tumorigenesis and personalized treatment of Chinese glioma population.

\section{INTRODUCTION}

The 2016 World Health Organization (WHO) classification of tumors of the central nervous system (CNS) has successfully updated in May, which promoted the pathology into a molecular era as the result of integration of relevant biomarkers [1]. The 2016 CNS WHO remarkably redefined the diffuse gliomas based on the molecular parameters and traditional histology features. It has not only added newly recognized neoplasms, but also deleted some entities that no longer have diagnostic and/or biological relevance. Most notably, the oligoastrocytoma, a diagnostic category suffered from high interobserver discordance in traditional pathology definition [2-4], could been pertinently reclassified into astrocytoma or oligodendroglioma according to the IDH and $1 p / 19 q$ status. All these indicated that the component and proportion of diffuse gliomas has virtually changed.

Considering the nonnegligible impact on component and proportion of glioma brought about by the 2016 CNS WHO, more comprehensive re-understanding of the epidemiological characteristics of "new" gliomas in 
adults appears to be particularly important. At the same time, more studies and clinic works should be carried on to investigate the reliability and validity of the new pathologic classification scheme.

Therefore, in the present study, we retrospectively reviewed 1210 adult patients with supratentorial gliomas in our institution and then reclassified their samples according to the 2016 CNS WHO. In addition, the epidemiological characteristics of these new pathology entities, including age at diagnosis, gender ratio, location and survival time were analyzed.

\section{RESULTS}

\section{Incidence of the newly diagnosed entities}

In our dataset, the most common histologic type in adults with supratentorial lesion was IDH-wildtype GBM (27.0\%) (Figure 1). The incidence of grade II, III and IV gliomas was $40.0 \%, 29.0 \%$ and $31.0 \%$, respectively (Supplementary Figure S1). Meanwhile, the most prevalent molecular subtype was gliomas with IDH-wildtype $(47.0 \%)$, followed by IDH-mutant and $1 \mathrm{p} / 19 \mathrm{q}$-codelted $(31.0 \%)$, and trailed by IDH-mutant $(22.0 \%)$ according to the 2016 classification scheme (Supplementary Figure S2). Comparing with 2007 CNS WHO, the oligoastrocytoma was no longer served as an independent diagnostic entity and thereby reclassified into either astrocytoma or oligodendroglioma in the light of $1 \mathrm{p} / 19 \mathrm{q}$ and IDH detection results.

Notably, we found the incidence of GBM decreased. According to the 2007 CNS WHO criteria, there were $384(32.0 \%) \mathrm{GBM}_{2007}$ [including $298 \mathrm{GBM}$ and 86 glioblastomas with oligodendroglioma component (GBMO)] in our cohort. After the reclassification based on 2016 CNS WHO, only 374 (31.0\%) patients were diagnosed with $\mathrm{GBM}_{2016}$, including 44 IDH-mutant GBM and 330 IDH-wildtype GBM. The rest of 10 patients who harbored $1 \mathrm{p} / 19 \mathrm{q}$-codeleted and IDH-mutant were reclassified into $\mathrm{AO}$ (WHO grade III) (Figure 1 and
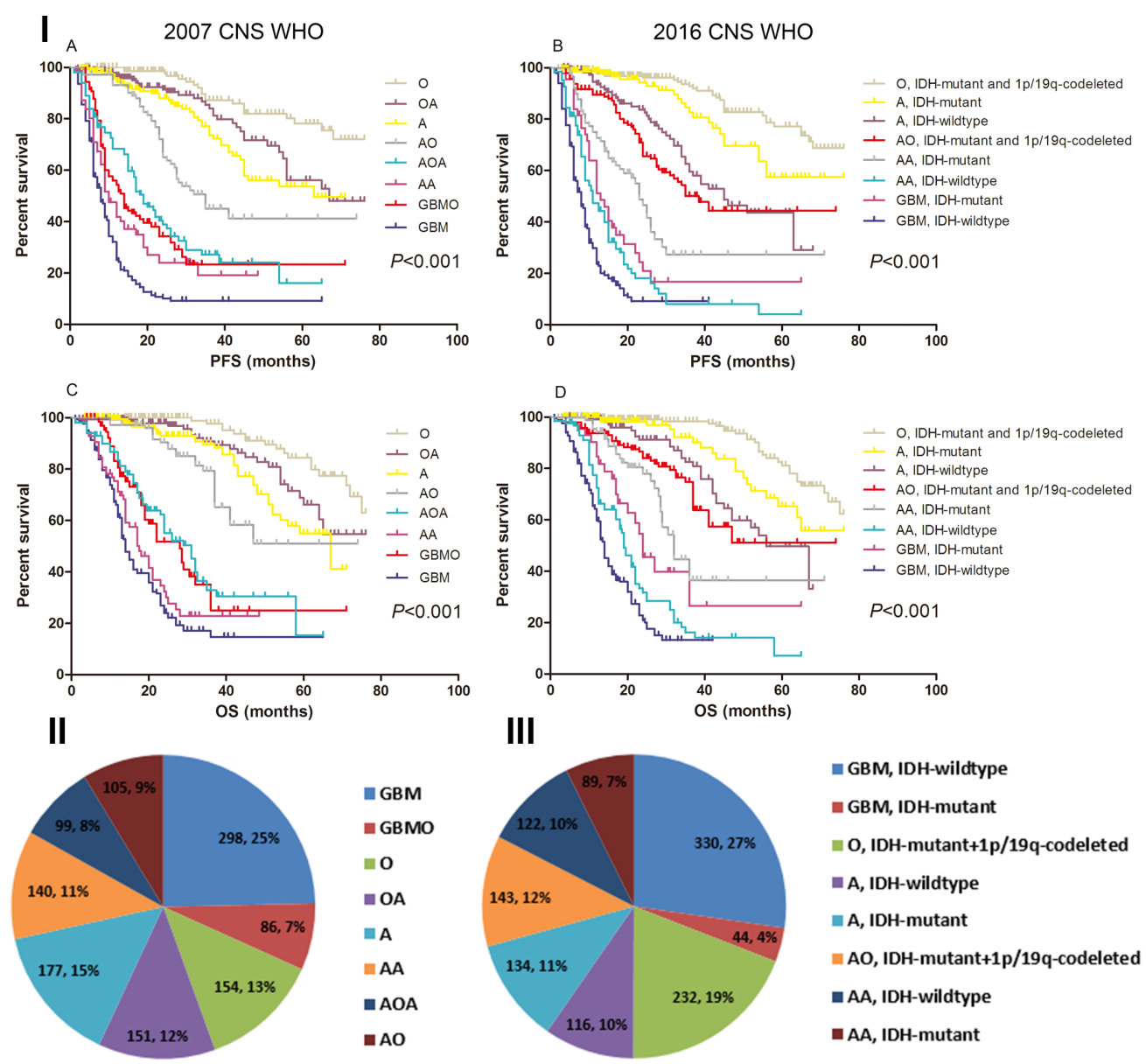

Figure 1: The survival rate and specific distribution of 2007/2016 CNS WHO pathology entities. I. Kaplan-Meier estimates of survival time in 2007/2016 CNS WHO pathology entities. Both the 2007 and 2016 CNS WHO could categorized patients into eight entities with different survival. II. Distribution of diagnostic entities in the 2007 CNS WHO (N=1210). GBM was the most frequently reported malignant tumor in the brain, which accounted for $25.0 \%$ of all the glioma. III. Distribution of diagnostic entities in the 2016 CNS WHO (N=1210). GBM with IDH-wildtype was the most common malignant tumor in the brain, which accounted for $27.0 \%$ of all the glioma. 
Table 1: Clinical characteristics of different glioma entities

\begin{tabular}{lccccc}
\hline Variables & $\begin{array}{c}\text { Number of } \\
\text { cases }\end{array}$ & $\begin{array}{c}\text { Median age at } \\
\text { diagnosis (years) }\end{array}$ & $\begin{array}{c}\text { Gender ratio } \\
\text { (M/F) }\end{array}$ & $\begin{array}{c}\text { Median PFS } \\
\text { (months) }\end{array}$ & $\begin{array}{c}\text { Median OS } \\
\text { (months) }\end{array}$ \\
\hline O, 1p/19q-codeleted and & 232 & 42 & $1.2: 1$ & N/A & N/A \\
IDH-mutant & 134 & 38 & $1.3: 1$ & N/A & N/A \\
A, IDH-mutant & 116 & 40.5 & $1.4: 1$ & 45.0 & 56.0 \\
A, IDH-wildtype & 143 & 42 & $2.5: 1$ & 38.5 & N/A \\
AO, 1p/19q-codeleted and & 89 & 43 & $1.9: 1$ & 23.0 & 32.0 \\
IDH-mutant & 122 & 42 & $1.8: 1$ & 11.0 & 19.0 \\
AA, IDH-mutant & 44 & 48 & $1: 1$ & 13.0 & 24.0 \\
AA, IDH-wildtype & 330 & 50 & $1.9: 1$ & 8.0 & 14.0 \\
GBM, IDH-mutant & & & & & \\
GBM, IDH-wildtype & & &
\end{tabular}

Abbreviation: PFS, progression-free survival; OS, overall survival; M, male; F, female; IDH, isocitrate dehydrogenase; O, oligodendroglioma; A, astrocytoma; $\mathrm{AO}$, anaplastic oligodendroglioma; $\mathrm{AA}$, anaplastic astrocytoma; GBM, glioblastoma multiforme; N/A, not available.

Supplementary Figure S1). Nevertheless, GBM was still the most frequently reported malignant tumor in the brain, which accounted for $31.0 \%$ of all the glioma (Figure 1).

\section{Relative survival of the newly diagnosed entities}

Overall, The higher grade, the shorter survival time $(P<0.001)$ (Supplementary Figure $\mathrm{S} 1)$. The patients concurrent with IDH-mutant and 1p/19q-codelted had the most favorable prognosis, while those with IDH-wildtype showed the shortest survival $(P<0.001)$ (Supplementary Figure S2). Interestingly, the Log-Rank analysis revealed that the IDH-mutant GBM showed a tendency of increased survival rate than IDH-wildtype AA, but the difference was not statistically significant [13.0 months vs. 11.0 months, $P=0.146$ for progression-free survival (PFS); 24.0 months $v s .19 .0$ months, $P=0.059$ for overall survival (OS)] (Figure 1). Furthermore, we have found that the median PFS and OS of $\mathrm{GBM}_{2016}$ were relatively shorter compared with $\mathrm{GBM}_{2007}(8.0$ months vs. 9.0 months and 14.0 months vs. 15.0 months) (Supplementary Figure S1).

\section{Distribution of gender and age of the newly diagnosed entities}

Almost all gliomas were more prevalent in males, although the gender difference were quite small in the case of WHO grade II gliomas (Table 1). The gender ratio was significantly higher in grade III gliomas compared with grade II or IV gliomas ( $P=0.008$, by Chi-Square) (Table 1). Besides, the incidence for male in IDH-wildtype GBM was 1.9 times higher than those in IDH-mutant GBM ( $P=0.05$, by Chi-Square) (Table 1$)$.

As for the age distribution, GBM with IDHwildtype was associated with the highest median age at diagnosis (median age $=50$ years old). The further subgroup comparison showed that the age at diagnosis was directly proportional to tumor grade ( $P=0.022$, by one-way ANOVA) (Table 1).

\section{Incidence by site and histology of the newly diagnosed entities}

The distribution of gliomas by site was shown in Figure 2. Patients concurrent with IDH-mutant and $1 \mathrm{p} / 19 \mathrm{q}$-codeleted $(47.0 \%)$ or with single IDH-mutant $(46.0 \%)$ were mainly distributed in frontal lobe, while those with IDH-wildtype (42.0\%) were dominant in temporal lobe (all $P<0.05$, by Chi-Square). The following subgroup analysis confirmed these findings in both astrocytic and oligodendroglial tumors.

Overall, frontal lobe accounted for $40.0 \%$, temporal $36.0 \%$, insular $4.0 \%$, and other brain $20.0 \%$ of all gliomas (Figure 2). The percentage of IDH-mutant and 1p/19qcodeleted oligodendroglioma $(\mathrm{O})$ in frontal lobe was $23.7 \%$, which was significantly higher than those of other pathologic types ( $P=0.001$, by Chi-Square). Similarly, the proportion of pathologic type in temporal lobe was driven by IDH-wildtype GBM (30.4\%), even though the P value was marginally significant ( $P=0.066$, by Chi-Square). In insular lobe, the most common pathologic type was IDH-mutant astrocytoma (A) $(29.6 \%)(P<0.001$, by ChiSquare).

Validation of the prognostic potential of the 2016 WHO classification system

Univariate analysis demonstrated that age $45 \geq$ years, gross total resection (GTR), karnofsky performance score (KPS) $\geq 70,1 \mathrm{p} / 19 \mathrm{q}$-codeleted, IDH- 
mutant, $\mathrm{O}^{6}$-methylguanine-DNA methyltransfer (MGMT) promoter Methylation and 2016 CNS WHO were associated with prognosis $(P<0.05$, data not shown). The 2016 CNS WHO categorized the whole cohort into eight subtypes with significantly distinct survival $(P<0.001)$ (Figure 1). In the further Cox proportional hazard model enrolled all these parameters, 2016 CNS WHO was confirmed as an independent factor affecting PFS and OS. The hazard ratio (HR) of the 2016 CNS WHO was 0.932 [95\% confidence interval $(\mathrm{CI}), 0.897-0.967 ; P<0.001]$ for PFS and 0.919 (95\% CI, 0.880-0.961; $P<0.001)$ for OS (Table 2).

\section{DISCUSSION}

Recently, the revised World Health Organization Classification of Tumors of the Central Nervous System has published on Acta Neuropathologica [1]. It brought several new perspectives towards traditional pathology: promote the development of pathology into a molecular era; acknowledge the existence tumor heterogeneity, especially in the biological phenotype; emphasize the role of molecular pathology in personalized treatment. In the Cox proportional hazard model, we have realized the robust potential of the new classification scheme in predicting prognosis. Since the 2016 classification system of gliomas has integrated with $1 \mathrm{p} / 19 \mathrm{q}$ and IDH status, the epidemiological characteristics of the new pathology entities also changed. Therefore, this study was designed to uncover the peak age incidence, gender ratio, tumor location and prognosis of the new entities.

In the present study, we found that the incidence and survival of $\mathrm{GBM}_{2016}$ has decreased. This situation was directly resulted from the fact that 10 GBMOs with 1p/19q-codeleted and IDH-mutant had been reclassified into grade III according to the 2016 diagnostic criteria.

\section{I $\mathrm{GBM}, \mathrm{IDH}$-wildtype}

a GBM, IDH-mutant

$\varpi 0, \mathrm{IDH}-$ mutant+1p/19q-codeleted $\square \mathrm{A}, \mathrm{IDH}$-wildtype

\section{A, IDH-mutant}

घAA, IDH-wildtype

\section{AO, IDH-mutant+1p/19q-codeleted}

\section{aA, IDH-mutant}
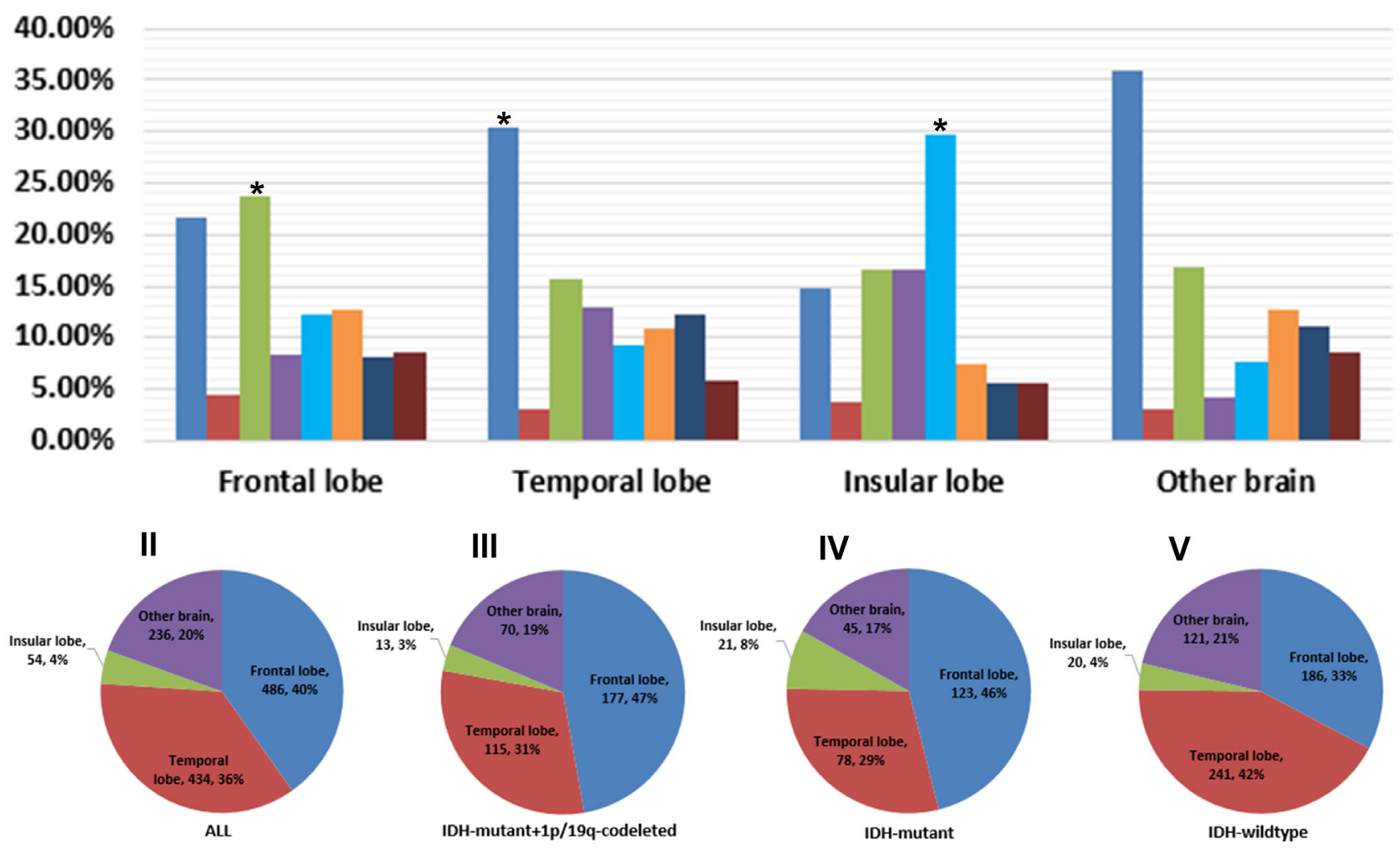

Figure 2: Regional constituents of pathological entities and preferential distribution of different tumor subtypes. I. Composing proportion of pathological entities in the frontal, temporal, insular and other brain sites. The predominant proportion in frontal, temporal and insular lobe was IDH-mutant and 1p/19q-codeleted O, IDH-wildtype GBM and IDH-mutant A, respectively. II-V. Almost $76.0 \%$ gliomas occurred in frontal and temporal lobe. Patients concurrent with IDH-mutant and 1p/19q-codeleted or with single IDHmutant were mainly distributed in frontal lobe, while those with IDH-wildtype were dominant in temporal lobe. 
Table 2: Multivariate analysis to identify factors that predict survival in all patients

\begin{tabular}{lccc}
\hline Variables & Hazard Ratio (HR) & 95\% Confidence Interval (CI) & P value \\
\hline Factors associated with PFS & & & \\
Age $\geq 45$ & 1.333 & $1.129-1.574$ & 0.001 \\
2016 WHO CNS & 0.932 & $0.897-0.967$ & $<0.001$ \\
1p/19q-codeleted & 0.432 & $0.323-0.578$ & $<0.001$ \\
IDH-mutant & 0.403 & $0.317-0.512$ & $<0.001$ \\
MGMT-Methylated & 0.748 & $0.616-0.907$ & 0.003 \\
GTR & 0.789 & $0.659-0.945$ & 0.010 \\
Factors associated with OS & & & \\
Age $\geq 45$ & 1.269 & $1.047-1.538$ & 0.015 \\
2016 WHO CNS & 0.919 & $0.880-0.961$ & $<0.001$ \\
1p/19q-codeleted & 0.421 & $0.295-0.602$ & $<0.001$ \\
IDH-mutant & 0.354 & $0.265-0.473$ & $<0.001$ \\
MGMT-Methylated & 0.793 & $0.634-0.990$ & 0.041 \\
GTR & 0.694 & $0.565-0.853$ & 0.001 \\
\hline
\end{tabular}

Abbreviation: HR, hazard ratio; 95\% CI, 95\% confidence interval; PFS, progression-free survival; OS, overall survival; WHO, world health organization; CNS, central nervous system; IDH, isocitrate dehydrogenase; MGTM, $\mathrm{O}^{6}$-methylguanineDNA methyltransfer; GTR, gross total resection;

It reduced the total number of WHO grade IV gliomas to some degree. However, GBM was still the most common malignant tumor in the brain, which accounted for $31.0 \%$ of all the glioma in our study. GBMO, as its name suggested, was glioblastomas with oligodendroglioma component, which implied decreased malignancy and relatively favorable prognosis [5-8]. Undoubtedly, the deletion of GBMO would attributed to shortened survival of patients with GBM over the next few years.

With one interesting exception, almost all new pathology entities in our study with survival trend followed the principle below: the higher grade, the shorter survival. We noted that patients with IDH-wildtype anaplastic astrocytoma (AA) exhibited worse prognosis than IDHmutated GBM, which was in accordance with the results reported by Hartmann et al. [9]. Our data showed the median OS of IDH-wildtype AA was 19.0 months, which was close to the survival of newly diagnosed "classic" GBM [10-12]. With this regard, it seemed to be more rational to group IDH-wildtype AA into WHO grade IV which implied more aggressive treatments were needed in routine clinical course.

In line with previous studies [13, 14], almost all gliomas were more frequent in male than female. In 2015, CBTRUS statistical report of primary brain tumors revealed that approximately $55.0 \%$ of the malignant tumors occurred in males $(65,056$ tumors) and $45.0 \%$ in females (51,967 tumors) [14]. This gender ratio was mainly driven by the most common brain malignant tumor-glioma. In the present study, the male-preference was particularly prominent in the cluster of WHO grade III gliomas and IDH-wildtype GBM. A statistical report from West China Glioma Center demonstrated there was a dramatically high incidence of malignant tumor in males [15]. But the mechanisms behind this association were still unclear. Perhaps it was correlated with the phenomenon that male was the major labor force in China and thereby had more exposure chance to harmful substance which signified higher risk for brain neoplasm [16].

In this study, GBM, the most malignant brain tumor, was correlated with the highest median age at diagnosis. GBM was primarily diagnosed at older ages, with higher rates between 75 and 84 years old [17]. Our data showed the median age at diagnosis of IDH-mutant GBM and IDH-wildtype GBM was only 48 years and 50 years, respectively, which was similar with result (51 years) provided by Wang et al. [15]. It's well-grounded that older age was a risk factor and always conferred to shorter survival [18-20]. Therefore, we ascertained that older age was a major reason for the dismal prognosis of patients with GBM.

The majority of gliomas occurred in the frontal and temporal lobes in our dataset which was in accordance with previous studies [14, 15]. We further found that gliomas concurrent with IDH-mutant and $1 p / 19 q-$ codeleted or with single IDH-mutant were mainly 
distributed in frontal lobe, while those with IDH-wildtype were prevailed in temporal lobe. It was consistent with our report in 2012 [21].

In the following subgroup analysis, we noted that oligodendroglioma with IDH-mutant and 1p/19qcodeleted was the most common tumor in frontal lobe. Mueller et al. reported the frequency of $1 p / 19 q$ codeletion of oligodendroglial tumors in the temporal lobe $(23.1 \%)$ was significantly less than that in non-temporal lobes (81.7\%) [22]. Furthermore, Huang et al., in 2008, found that oligodendroglial tumors located in the nontemporal lobes were significantly more likely to have combined deletion of $1 p$ and $19 q$ compared with tumors arising in the insula, temporal lobe, and temporal with another lobe $(P=0.001)$ [23]. It's well-established that oligodendroglioma with $1 \mathrm{p} / 19 \mathrm{q}$-codeleted always had better response to procarbazine, lomustine, and vincristine (PCV) chemotherapy regimen [24-26]. Therefore, in the under-developed areas without resource to detect the $1 p / 19 q$ status, $P C V$ protocol remained a relatively reasonable alternative for patients with gliomas located in frontal lobe.

Interestingly enough, we noticed primary GBM occupied absolute predominance in temporal lobe which was characterized by low incidence of IDH mutation and dismal prognosis. This finding was consistent with previous research results [21, 27]. While gliomas of insular origin were more likely to be low-grade gliomas, such as IDH-mutant astrocytoma, but were less likely to be glioblastoma and anaplastic astrocytoma. The results implied that gliomas located in different lobes might confer to distinct gene phenotypes, which could provide new insights into potential overlap between different prognostic variables and might help to identify niche locations for glioma cells of origin [28]. But unfortunately, the underlying molecular mechanism of these phenomena still remains unclear.

There are also some limitations in the current study. First, it's a single-institution study. Our department is committed to treating patients with supratentorial lesions and aged from 16 years to 60 years, which will lead to bias, especially in analysis of age at diagnosis. Second, diffuse midline glioma is a new and very important entity in the 2016 WHO Classification of Tumors of the CNS. However, it's not included in this manuscript because of the relatively small sample. Third, the median PFS and OS of several subgroups are not available because of the relatively short follow-up. Therefore, in the future, we will continue our study with long-term follow-up in order to revalidate these results.

In conclusion, the 2016 CNS WHO classification scheme is an independent factor associated with prognosis. This study elucidates the epidemiological characteristics as well as the regional constituents of these new gliomas entities, which could bring insights into tumorigenesis and personalized treatment of Chinese glioma population.

\section{PATIENTS AND METHODS}

\section{Patients and tumor samples}

Records from a consecutive series of 1210 patients with a histological diagnosis of gliomas and complete clinical data from January 2009 to May 2016 were retrospectively enrolled in the present study. All specimens were independently reviewed by 3 experienced neuropathologists (Jumei Wang, Guang Li, and Lin Luo), who were blinded to the clinical outcomes of the patients, according to the 2007 and 2016 CNS WHO criteria [1, 29]. This study was approved by the institutional review board of Capital Medical University. All the participants have provided informed consent for this study.

Tumor location usually refers to the lobe or region of the brain, in which the bulk of the tumor resided. They were specified according to the International Classification of Diseases, version 10 (ICD-10) as previously described by Larjavaara [30]. Besides, we added another location: insular lobe, according to the classification of tumors of limbic and pralimbic systems by Yasargil [31]. The orientation was judged by the MR images, including T1weighted axial, coronary, and sagital images pre- and postenhancement and T2-weighted axial images. Progressionfree survival (PFS) was designated as the time from the first operation to the time of tumor recurrence. Overall survival (OS) was calculated from the date of surgical resection until death or the last known follow-up. The resection degree was confirmed by postoperative contrastenhanced MRI within 48-72 hours. All patients enrolled in the present study were treated according to the latest National Comprehensive Cancer Network guideline. The specific treatment protocol has been elaborated detailedly in our previous study [32].

\section{Evaluation of 1p/19q, IDH and MGMT}

$1 \mathrm{p} / 19 \mathrm{q}$ abnormality was determined by fluorescence in situ hybridization (FISH) with 1 p36/1q25 or 19q/19p locus-specific identifier DNA dual color probes (Vysis). The experiment protocol and interpretation principle have been elaborated in a previous study [32]. For each probe, $>100$ nonoverlapping nuclei were enumerated per hybridization. Tumors with more than $30 \%$ of nuclei showing DNA loss were defined as tumor with chromosomal loss. MGMT promoter methylation status was evaluated by methylation specific PCR (MSP) and IDH mutation was detected by sequence analysis, as described in another paper from our team [33].

\section{Statistical analysis}

One-way ANOVA was employed to compare mean age between different groups. Categorical variables were compared using the chi-square test or Fisher exact test, where appropriate. Survival analysis was calculated by the 
Kaplan-Meier method and group results were compared using the Log-rank test. Multivariate analysis to identify independent prognostic factors was carried out using the Cox proportional hazards model. All statistical analyses were performed with SPSS (version 19.0, Chicago, IL, USA). For all tests, a $P$ value less than 0.05 was considered statistically significant.

\section{ACKNOWLEDGMENTS}

We acknowledge Dr. Lin Luo for pathology diagnosis, the pathology department, Beijing Neurosurgical Institute, and Dr. Guang Li, Pathology department, Beijing Tiantan Hospital, Capital Medical University.

\section{CONFLICTS OF INTEREST}

No potential conflicts of interest were disclosed.

\section{FUNDING STATEMENT}

We acknowledge financial support by National Natural Science Foundation of China (81571632), and the Capital Health Research and Development of Special (2014-2-2042).

\section{REFERENCES}

1. Louis DN, Perry A, Reifenberger G, von Deimling A, Figarella-Branger D, Cavenee WK, Ohgaki H, Wiestler OD, Kleihues P, Ellison DW. The 2016 World Health Organization Classification of Tumors of the Central Nervous System: a summary. Acta Neuropathol. 2016; 131:803-20.

2. Giannini C, Scheithauer BW, Weaver AL, Burger PC, Kros JM, Mork S, Graeber MB, Bauserman S, Buckner JC, Burton J, Riepe R, Tazelaar HD, Nascimento AG, et al. Oligodendrogliomas: reproducibility and prognostic value of histologic diagnosis and grading. J Neuropathol Exp Neurol. 2001; 60:248-62.

3. van den Bent MJ. Interobserver variation of the histopathological diagnosis in clinical trials on glioma: a clinician's perspective. Acta Neuropathol. 2010; 120:297-304.

4. Jiang H, Ren X, Cui X, Wang J, Jia W, Zhou Z, Lin S. $1 \mathrm{p} / 19 \mathrm{q}$ codeletion and IDH1/2 mutation identified a subtype of anaplastic oligoastrocytomas with prognosis as favorable as anaplastic oligodendrogliomas. Neuro Oncol. 2013; 15:775-82.

5. Kraus JA, Lamszus K, Glesmann N, Beck M, Wolter M, Sabel M, Krex D, Klockgether T, Reifenberger G, Schlegel U. Molecular genetic alterations in glioblastomas with oligodendroglial component. Acta Neuropathol. 2001; 101:311-20
6. Appin CL, Gao J, Chisolm C, Torian M, Alexis D, Vincentelli C, Schniederjan MJ, Hadjipanayis C, Olson JJ, Hunter S, Hao C, Brat DJ. Glioblastoma with oligodendroglioma component (GBM-O): molecular genetic and clinical characteristics. Brain Pathol. 2013; 23:454-61.

7. Miller CR, Dunham CP, Scheithauer BW, Perry A. Significance of necrosis in grading of oligodendroglial neoplasms: a clinicopathologic and genetic study of newly diagnosed high-grade gliomas. J Clin Oncol. 2006; 24:5419-26.

8. Vordermark D, Ruprecht K, Rieckmann P, Roggendorf W, Vince GH, Warmuth-Metz M, Kölbl O, Flentje M. Glioblastoma multiforme with oligodendroglial component (GBMO): favorable outcome after post-operative radiotherapy and chemotherapy with nimustine (ACNU) and teniposide (VM26). BMC Cancer. 2006; 6:247.

9. Hartmann C, Hentschel B, Wick W, Capper D, Felsberg J, Simon M, Westphal M, Schackert G, Meyermann R, Pietsch T, Reifenberger G, Weller M, Loeffler M, von Deimling A. Patients with IDH1 wild type anaplastic astrocytomas exhibit worse prognosis than IDH1-mutated glioblastomas, and IDH1 mutation status accounts for the unfavorable prognostic effect of higher age: implications for classification of gliomas. Acta Neuropathol. 2010; 120:707-18.

10. Kim BS, Seol HJ, Nam DH, Park CK, Kim IH, Kim TM, Kim JH, Cho YH, Yoon SM, Chang JH, Kang SG, Kim $\mathrm{EH}$, Suh CO, et al. Concurrent Chemoradiotherapy with Temozolomide Followed by Adjuvant Temozolomide for Newly Diagnosed Glioblastoma Patients: A Retrospective Multicenter Observation Study in Korea. Cancer Res Treat. 2017; 49:193-203.

11. Bhavsar S, Hagan K, Arunkumar R, Potylchansky Y, Grasu R, Dang A, Carlson R, Cowels C, Arnold B, Rahlfs TF, Lipski I, Walsh C, Nguyen AT, et al. Preoperative statin use is not associated with improvement in survival after glioblastoma surgery. J Clin Neurosci. 2016; 31:176-80.

12. Sacko A, Hou MM, Temgoua M, Alkhafaji A, Marantidou A, Belin C, Mandonnet E, Ursu R, Doridam J, Coman I, Levy-Piedbois C, Carpentier AF. Evolution of the Karnosky Performance Status throughout life in glioblastoma patients. J Neurooncol. 2015; 122:567-73.

13. Schwartzbaum JA, Fisher JL, Aldape KD, Wrensch M. Epidemiology and molecular pathology of glioma. Nat Clin Pract Neurol. 2006; 2:494-503; quiz 1 p following 516.

14. Ostrom QT, Gittleman H, Fulop J, Liu M, Blanda R, Kromer C, Wolinsky Y, Kruchko C, Barnholtz-Sloan JS. CBTRUS Statistical Report: Primary Brain and Central Nervous System Tumors Diagnosed in the United States in 2008-2012. Neuro Oncol. 2015; 17 Suppl 4:iv1-iv62.

15. Wang X, Chen JX, Zhou Q, Liu YH, Mao Q, You C, Chen N, Xiong L, Duan J, Liu L. Statistical Report of Central Nervous System Tumors Histologically Diagnosed in the Sichuan Province of China from 2008 to 2013: A West 
China Glioma Center Report. Ann Surg Oncol. 2016; 23:946-953.

16. Gomes J, Al Zayadi A, Guzman A. Occupational and environmental risk factors of adult primary brain cancers: a systematic review. Int J Occup Environ Med. 2011; 2:82-111.

17. Pereira AF, Carvalho BF, Vaz RM, Linhares PJ. Glioblastoma in the elderly: therapeutic dilemmas. Surg Neurol Int. 2015; 6:S573-82.

18. Babu R, Komisarow JM, Agarwal VJ, Rahimpour S, Iyer A, Britt D, Karikari IO, Grossi PM, Thomas S, Friedman AH, Adamson C. Glioblastoma in the elderly: the effect of aggressive and modern therapies on survival. J Neurosurg. 2016; 124:998-1007.

19. Chaichana K, Parker S, Olivi A, Quiñones-Hinojosa A. A proposed classification system that projects outcomes based on preoperative variables for adult patients with glioblastoma multiforme. J Neurosurg. 2010; 112:997-1004.

20. Brodbelt A, Greenberg D, Winters T, Williams M, Vernon S, Collins VP, and (UK) National Cancer Information Network Brain Tumour Group. Glioblastoma in England: 2007-2011. Eur J Cancer. 2015; 51:533-42.

21. Ren X, Cui X, Lin S, Wang J, Jiang Z, Sui D, Li J, Wang Z. Co-deletion of chromosome $1 \mathrm{p} / 19 \mathrm{q}$ and IDH1/2 mutation in glioma subsets of brain tumors in Chinese patients. PLoS One. 2012; 7:e32764.

22. Mueller W, Hartmann C, Hoffmann A, Lanksch W, Kiwit J, Tonn J, Veelken J, Schramm J, Weller M, Wiestler OD, Louis DN, von Deimling A. Genetic signature of oligoastrocytomas correlates with tumor location and denotes distinct molecular subsets. Am J Pathol. 2002; 161:313-19.

23. Huang L, Jiang T, Yuan F, Li GL, Liu EZ, Wang ZC. Correlations between molecular profile and tumor location in Chinese patients with oligodendroglial tumors. Clin Neurol Neurosurg. 2008; 110:1020-24.

24. Taal W, van der Rijt CC, Dinjens WN, Sillevis Smitt PA, Wertenbroek AA, Bromberg JE, van Heuvel I, Kros JM, van den Bent MJ. Treatment of large low-grade oligodendroglial tumors with upfront procarbazine, lomustine, and vincristine chemotherapy with long follow-up: a retrospective cohort study with growth kinetics. J Neurooncol. 2015; 121:365-72.

25. Stege EM, Kros JM, de Bruin HG, Enting RH, van Heuvel I, Looijenga LH, van der Rijt CD, Smitt PA, van den Bent MJ. Successful treatment of low-grade oligodendroglial tumors with a chemotherapy regimen of procarbazine, lomustine, and vincristine. Cancer. 2005; 103:802-09.

26. van den Bent MJ, Brandes AA, Taphoorn MJ, Kros JM, Kouwenhoven MC, Delattre JY, Bernsen HJ, Frenay M, Tijssen CC, Grisold W, Sipos L, Enting RH, French PJ, et al. Adjuvant procarbazine, lomustine, and vincristine chemotherapy in newly diagnosed anaplastic oligodendroglioma: long-term follow-up of EORTC brain tumor group study 26951. J Clin Oncol. 2013; 31:344-50.

27. Zada G, Bond AE, Wang YP, Giannotta SL, Deapen D. Incidence trends in the anatomic location of primary malignant brain tumors in the United States: 1992-2006. World Neurosurg. 2012; 77:518-24.

28. Ellingson BM, Lai A, Harris RJ, Selfridge JM, Yong WH, Das K, Pope WB, Nghiemphu PL, Vinters HV, Liau LM, Mischel PS, Cloughesy TF. Probabilistic radiographic atlas of glioblastoma phenotypes. AJNR Am J Neuroradiol. 2013; 34:533-40.

29. Louis DN, Ohgaki H, Wiestler OD, Cavenee WK, Burger PC, Jouvet A, Scheithauer BW, Kleihues P. The 2007 WHO classification of tumours of the central nervous system. Acta Neuropathol. 2007; 114:97-109.

30. Larjavaara S, Mäntylä R, Salminen T, Haapasalo H, Raitanen J, Jääskeläinen J, Auvinen A. Incidence of gliomas by anatomic location. Neuro Oncol. 2007; 9:319-25.

31. Yaşargil MG, von Ammon K, Cavazos E, Doczi T, Reeves JD, Roth P. Tumours of the limbic and paralimbic systems. Acta Neurochir (Wien). 1992; 118:40-52.

32. Jiang $\mathrm{H}$, Ren X, Zhang Z, Zeng W, Wang J, Lin S. Polysomy of chromosomes 1 and 19: an underestimated prognostic factor in oligodendroglial tumors. J Neurooncol. 2014; 120:131-38.

33. Jiang H, Ren X, Wang J, Zhang Z, Jia W, Lin S. Shortterm survivors in glioblastomas with oligodendroglioma component: a clinical study of 186 Chinese patients from a single institution. J Neurooncol. 2014; 116:395-404. 